\title{
Inexpensive Immersive Environments
}

\author{
Jeffrey Hole \\ Rochester Institute of Technology \\ One Lomb Memorial Drive \\ Rochester, NY 14623-5603 \\ jdhole@gmail.com
}

\author{
Jon Schull \\ Rochester Institute of Technology \\ One Lomb Memorial Drive \\ Rochester, NY 14623-5603 \\ 001.585.738.6696 \\ jis@it.rit.edu
}

\begin{abstract}
For adults, immersive environments for collaboration and play are expensive computer-intensive novelties. For children, they are commonplace but computer-free: kindergarten classrooms and playgrounds. Kids know how to develop, and spontaneously learn from imaginary play. If children were provided with robust, intuitive, and inexpensive computer-enabled environments they would thrive, learn, and engage in creative play, not just in physical and social worlds, but within increasingly important virtual worlds. We have developed a potentially ubiquitous, robust, intuitive, and cheap computer-enabled immersive environment for children, and have designed interactive technology that can be configured to support and enhance, rather than to structure and constrain children's play.
\end{abstract}

\section{Categories and Subject Descriptors}

H.5.2 [User Interfaces]: Screen Design ; H.5.3 [Group and Organization Interfaces]: Collaborative Computing; K.3.1 [Computer Uses in Education]: Collaborative Learning

\section{General Terms}

Design, Economics, Experimentation, Human Factors

\section{Keywords}

Immersive environment, children, collaborative play, participatory design

\section{INTRODUCTION}

For adults, immersive environments for collaboration and play are expensive computer-intensive novelties. For children, they are commonplace, but computer-free: kindergarten classrooms and playgrounds.

We have explored an inexpensive approach to immersive computing that could potentially expand the market for immersive technologies to new markets, including children, schools, and living rooms. Children normally develop their own immersive

Permission to make digital or hard copies of all or part of this work for personal or classroom use is granted without fee provided that copies are not made or distributed for profit or commercial advantage and that copies bear this notice and the full citation on the first page. To copy otherwise, to republish, to post on servers or to redistribute to lists, requires prior specific permission and/or a fee. IMMERSCOM 2009, May 27-29, Berkeley, USA

Copyright (C) 2009 978-963-9799-39-4

DOI 10.4108/immerscom.2009.20 environments using their imaginations during collaborative play. Although most imaginary play occurs spontaneously, researchers have explored technologies that foster collaborative story creation and play $[1,2,3,5,7,8]$. In this paper we also describe a novel and affordable immersive application that extends collaborative play by allowing children to navigate a digital story-land and create their own narratives. Finally, we describe an interface that unobtrusively fosters interaction and collaboration among users, and potentially enhances social learning and development.

The systems described here were designed and implemented over the course of a 10 week class on Innovation and Invention at RIT. Although they are prototypes, and while we have not yet tested our digital content with the children for whom it was designed, we believe the system shows considerable promise.

\section{DESIGN REQUIREMENTS AND MOTIVATIONS}

We envisioned a multi-user space through which children could collaboratively experience and explore a shared immersive environment. Our requirements for the system were as follows:

Economical: The system must be feasible for school districts to purchase or create using widely available, reasonably priced technology.

Simplistic Construction: The system must be easily and quickly assembled and disassembled.

Scalability: The navigation system should work with one to thirty students.

Untethered Navigation: The technology must allow users to move freely within an immersive environment and allow children to easily navigate a digital world.

Encouraging Collaboration: All users should be able to participate in navigation decisions. The system should reward increasing collaboration.

We thus sought a system that could be implemented anywhere within a school, provide children with experiences and freedom of movement not otherwise available, and allow them to play and collaborate in a natural manner.

\section{IMPLEMENTATION}

The resulting prototype has three parts: the immersive environment, the collaborative navigation system, and the virtual content. Each part can be used independently, but together they satisfy our requirements and motivations. 


\subsection{Immersive Environment}

The immersive environment was constructed of software, hardware, "tentware", and digital content. Many kinds of digital content are compatible with our system, but we decided that a three-dimensional digital world populated by storybook landmarks from children's literature would offer children an exceptional experience in an immersive environment. As a result, we focused on software tools for creating 3-D worlds, and used Google SketchUp and Google Earth as content development tools. By navigating a geographical location in Google Earth, users could trigger an immersive adaptation of Google SketchUp. Specifically, when users found their way to Rochester Institute of Technology via Google Earth, a storybook world created in Sketchup popped up and surrounded visitors on all sides. As described below, this 360 degree, 3 dimensional fantasy world was composed of forests, homes, palaces, and different paths for exploration along with clues and road signs to encourage social problem solving and interaction.

The physical attributes of the environment were created through the use of a white fabric tent-like dome and four digital projectors. Tent construction was similar to that of an outdoor backpacking tent reinforced with fiberglass meridians. Upon construction, the dome stood approximately nine feet high and had a diameter of eight feet at its widest point.

Python and ruby scripts initialized and ran four copies of Google Sketchup on a single computer. The scripts also pointed each copy's virtual camera in a different cardinal direction, synchronized their movements through the virtual world, and positioned their windows on the computer's desktop. By arranging the four projectors around the dome and connecting them to inexpensive triple head graphics boxes, which shunt the four Sketchup windows to the four projectors, we were able to encompass users within a navigable virtual world.

\subsection{Collaborative Interaction}

Navigation of the digital world was achieved using image processing technology. A web camera was mounted at the top of the dome and pointed downward to feed overhead images of the users into a nearby computer. Image processing software and network-enabled scripts were used to filter these images, compute a "center of gravity" and use that to generate virtual cursor movements. The users' movement within the dome were thus tracked and used to create corresponding movement commands in the virtual world, so that when users moved toward the North edge of the dome, the virtual cameras moved smoothly "Northward" in the virtual world.

Initially, there were a number of difficulties in tracking users and their movement. First, web cameras have fairly limited viewing angles, restricting our ability to track the full body of the user. At the extreme edges of the dome, a user's feet were the only indicators of a their presence. Furthermore it was sometimes hard to distinguish the user from the background (a tiled floor). We addressed these challenges with two techniques, each of which was moderately successful.

In the first case, users were given directional infrared lights to carry when inside the dome. The video software then identified user location by tracking the infrared light. The second method was to cover the floor with white material to heighten contrast. Users could then enter the dome without any additional equipment. This created an environment in which the users could relax and move naturally.

When thus configured and oriented correctly, the system computed a visual "center of gravity" that tracked the people in the dome (Figure 1). For example, if four individuals were equally spaced at the edges of the dome, the center of gravity was at the middle of the dome and no movement commands would be

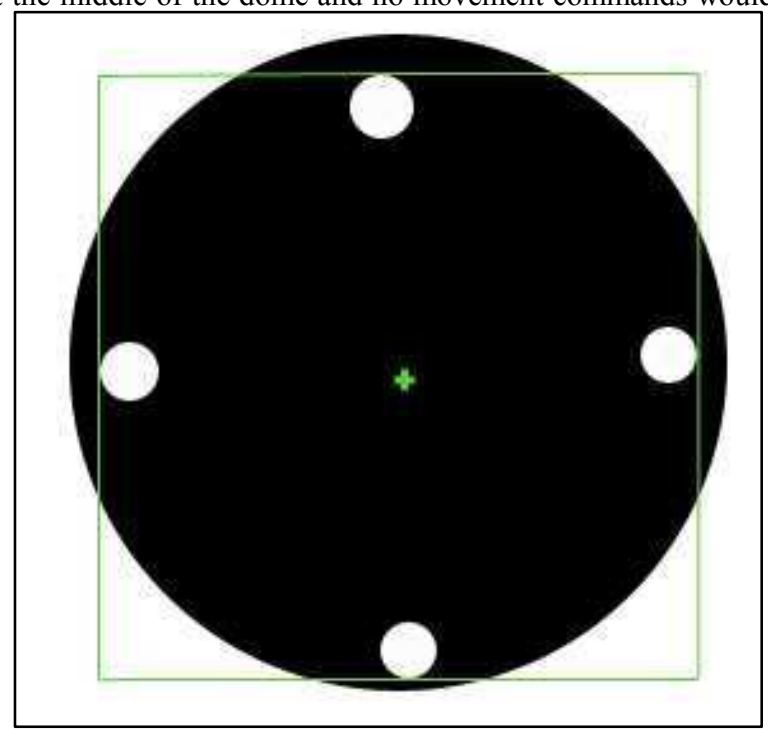

Figure 1: An example view of the inside of the dome after filtering and determining center of gravity.

registered. However, if one or more individuals shifted position, the center of gravity would shift, and movement would begin. When users move back to the center of the dome or disperse, the center of gravity shifts to the middle of the dome and movement subsides.

\subsection{Fantasy World}

As illustrated in Figures 2 and 3, we used Google Sketchup to create a virtual environment in which children could immersively experience fantasy objects they would recognize from traditional fairytales.

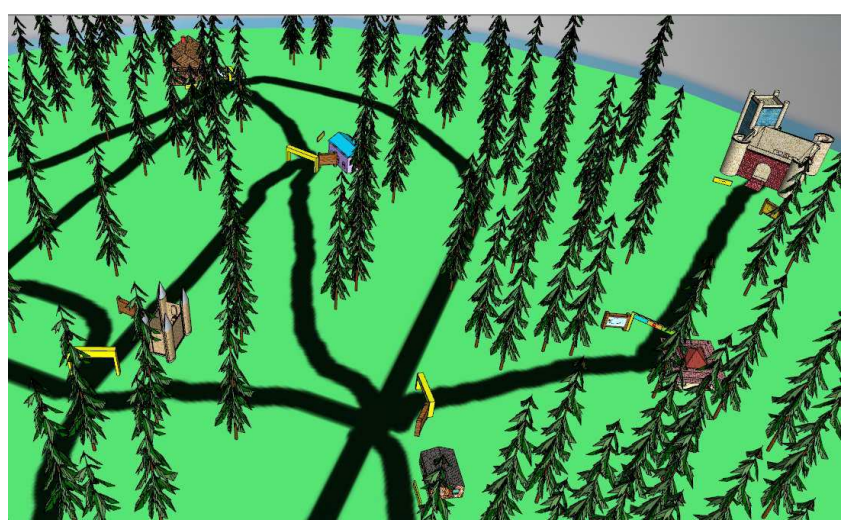

Figure 2: Aerial view of the fantasy world with the view of castles and cabins

The 3-D world was composed of a variety of landscapes. Users could wander through forests, ponds, and meadows. The world 
also included a number of cabins, houses, workshops, and castles. Each structure was three dimensional, allowing users to walk under arches, through doors, and explore inside buildings.

Each of the virtual buildings could be reached by following marked paths introduced so that users would not become lost, but would remain free to wander through the landscape as the desired.

In order to explore the educational and recreational potential of this system, the fantasy world also included a number of billboards which provided clues and directions when users got close enough to read them (Figure 3). Some clues provided details on the destination of each path; others required that

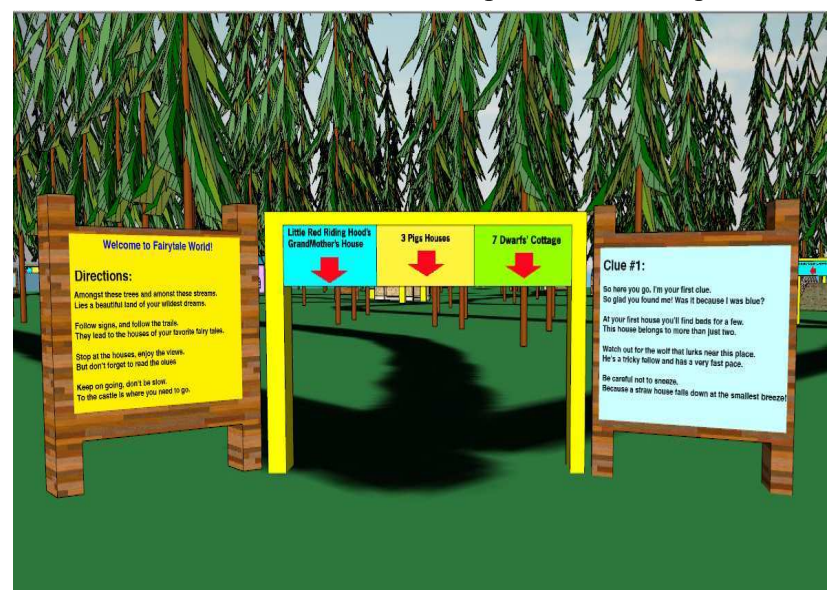

Figure 3: Billboards providing clues and directions in fantasy world

children use classroom knowledge, such as adding or subtracting, to match each path to a given clue.

\section{DISCUSSION}

\subsection{Related Work}

Previous research has sought to use technology to foster story creation and story navigation. Most of this research details experiments with technologies that allow children to develop, explore and narrate new stories. Among these, KidPad works with a traditional computer interface but extends it to support collaboration by providing multiple mice. StoryMat and StoryRooms take collaborative interactions a step further with unique tangible interfaces, which can be used by children to create content. Lastly, the Magic Carpet and KidsRoom build upon content creation technology to focus on digital story navigation.

KidPad enhances collaborative story creation by allowing two or three children to simultaneously co-create a digital story [3]. Multiple mice let children can draw, zoom, and link drawn scenes together to create a story, using a simple, easy to use interface. However, KidPad is restricted to a few users and places them in a traditional deskbound posture in front of a keyboard, mouse, and screen.

StoryMat uses tangible interfaces for greater creativity. Children move wirelessly connected stuffed animals around on a soft play mat that records the children's narrations and the toy's movements [5]. When another child visits the mat, the previous child's story is retrieved, for the new child to build upon or replace entirely [5]. The StoryMat thus offers the child an imaginary playmate and coordinates asynchronous collaboration among successive users [5].

Researchers at University of Maryland have also utilized tangible interfaces to create "storyrooms" [1] where children can act out stories and create new ones based on interactions with various wirelessly connected items scattered throughout an entire room. Although this design allows a classroom of children to experience the story and act it out, the room posed a number of problems. Because designing tangible interfaces for content that had not yet been created was particularly perplexing [1], the investigators to a standard story around which interfaces and slight variations could be more easily created.

While story creation is thus an area of active inquiry, navigation of digital stories has been less thoroughly explored [7]. The University of Nottingham's Mixed Reality Lab extended KidPad with a "magic carpet" in the form of a rectangular mat with pressure pads under each of the four sides [8]. By walking on the mat, children can zoom and pan to explore a drawing created with KidPad. Although this technology doubles the number of children who can collaborate using KidPad it is limited by floor space and the costs of the pressure pads. The system was also designed for the navigation of a two-dimensional picture rather than a continuous and interactive digital story.

Finally, KidsRoom used six computers and three cameras to track children as they moved about a room [2]. The room was set up with controlled lighting and a grey covered floor to facilitate tracking. The children interacted with pre-defined story script that required them to interact with various objects in the room. For example, children were to hide behind a bed when they heard growling, or talk to a clothes bureau that would respond to their requests [2].

Although KidsRoom thus let children fully participate in the story, it required a specialized space, a considerable amount of specialized hardware, and a fully scripted story. When children performed actions outside the system's expectations, system administrators had to intercede [2].

Thus, previous approaches to child-created content and story navigation have allowed small groups of children to interact with special software in specialized environments. Interactions tend to be scripted with a linear storyline, forcing children to follow along an adult determined path. In contrast our system can accommodate larger numbers of children in an environment comprised of inexpensive fabric walls, off-the-shelf hardware, and a digital world in which navigation is spatially and sequentially open, supported but not constrained by paths and signposts.

To our knowledge, this is the lightest and cheapest multi-projector virtual reality system that has been described in the literature. The closest comparison would seem to be Jacobson's CaveUT, which uses a modified version of the game Unreal Tournament 2004 to create an immersive environment [4].

Unlike our setup which uses a single computer to drive up to six displays, CaveUT uses one master computer and an additional computer for each of up to 31 displays. The advantages of a fully developed game engine are many; beautiful 3D content is already available to display and additional content can easily be built with the provided editing tools. However, our system is far more adaptable, and can both create and display content using a variety of off-the-shelf applications, including 3D games, panoramic 
images and movies, and architectural models. For example, the content creation tools in Google Sketchup, and Director proved useful for creation and rendering navigable interactive 3D models. Looking ahead, we have begun designing radically simple content creation tools to be built on top of Adobe's Director that would transform a user-created 2D map into a 3D environment, converting symbols into pre-built models, lines into roadways, and gray-scale gradients into rolling hills. This would allow children, to build 3D immersive environments with minimal training. This system would also let us have animated vehicles driving on the roads and emitting surround sound for added immersion.

\subsection{Results}

Although we have not yet tested our system with children, initial observations of students, faculty, and other adults interacting with the system have been positive, and suggest that the immersive projection and interaction methods have real promise.

Most previous work on digital story creation and interaction relied on a traditional computer screen or a single digital projector. All users see the same screen and a scene that can be encompassed in a single field of view. In the immersive environment (as in life), a single user can not observe all of the surrounding digital content as they move through the digital world. This seems to encourage collaborative interaction as multiple users distribute attention around the dome and share their observations with each other.

Thus, as users moved towards a virtual destination, they would periodically stop and spread out inside the dome, each user examining a different part of the three hundred and sixty degree landscape. Discussions ensued, with users comparing notes on how their portion of the screen fit into the larger scene.

Collaborative interaction was also promoted by the structure of the fantasy world. Users would move collaboratively to approach the billboards scattered throughout the virtual environment, read through the clues, pool suggestions on the next path to take, discuss and organize their thoughts before proceeding to the next destination.

It does seem plausible that this paradigm would enhance children's social, linguistic and perhaps even motor skills as they work as a group to apprehend the world, proceed with the digital story, and move through the physical and virtual environments without stepping on each other's toes.

\section{FUTURE OPPORTUNITIES}

Many other application domains within schools and without could probably benefit from a lightweight and inexpensive immersive environments.

For example, standard history lessons given to children and young adults often consist of readings, discussions, and video reenactments. However, for some children these methodologies fail to bring the material to life and as a result do not engage the child. With an immersive environment, educators can have students step inside historical events, and wander through their settings.

Outside of the classroom, interior designers, architects, landscapers, and home furnishing stores could let customers, unencumbered by virtual reality headgear, select and experience color schemes, furniture arrangements, and building layouts, all under simulated lighting conditions. Surgeons could explore and explain their procedures in non-threatening immersive environments. Law enforcement officials could visit the crime scene as it would have appeared at the time of the crime. This extends to the courtroom, where prosecutors could provide jurors with an immersive visit to the crime scene. Panoramic home movies displayed in immersive home entertainment environments could "offer trips down memory lane" to millions of consumers.

In the last few years, powerful and inexpensive computers, projectors, software packages have made mainstream immersive computing possible. Simple systems with broad utility and appeal could make it actual. Children who grow up with those systems will inevitably re-conceptualize the whole space, hopefully with newly-unfettered imaginations.

Our goal is not to rival or diminish the richness of the child's imagination but to provide a new set of "props" and options for the theater of the mind in an interesting, affordable, flexible digital environment.

\section{ACKNOWLEDGMENTS}

Special thanks to the entire Innovation and Invention class in the spring trimester of 2007. Each class member contributed to the implementation of this project.

\section{REFERENCES}

[1] Alborzi, H., Druin, A., Montemayor, J., Platner, M., Porteous, J., Sherman, L., Boltman, A., Taxén, G., Best, J., Hammer, J., Kruskal, A., Lal, A., Schwenn, T. P., Sumida, L., Wagner, R., and Hendler, J. 2000. Designing

StoryRooms: interactive storytelling spaces for children. In Proceedings of the Conference on Designing interactive Systems: Processes, Practices, Methods, and Techniques (New York City, New York, United States, August 17 - 19, 2000). D. Boyarski and W. A. Kellogg, Eds. DIS '00. ACM Press, New York, NY, 95-104. DOI= http://doi.acm.org/10.1145/347642.347673

[2] A. Bobick and et al. The kidsroom: A perceptually-based interactive and immersive story environment. Technical Report 398, MIT Media Lab Perceptual Computer Section, 1996. DOI= http://citeseer.ist.psu.edu/article/bobick99kidsroom.html

[3] Hourcade, J. P., Bederson, B. B., Druin, A., and Taxén, G. 2002. KidPad: collaborative storytelling for children. In $\mathrm{CHI}$ '02 Extended Abstracts on Human Factors in Computing Systems (Minneapolis, Minnesota, USA, April 20 - 25, 2002). CHI '02. ACM Press, New York, NY, 500-501. DOI= http://doi.acm.org/10.1145/506443.506449

[4] Jacobson, J., Le Renard, M., Lugrin, J., and Cavazza, M. 2005. The CaveUT system: immersive entertainment based on a game engine. In Proceedings of the 2005 ACM SIGCHI international Conference on Advances in Computer Entertainment Technology (Valencia, Spain, June 15 - 17, 2005). ACE '05. ACM Press, New York, NY, 184-187. DOI= http://doi.acm.org/10.1145/1178477.1178503

[5] Ryokai, K. \& Cassell, J. (1999). Computer Support for Children's Collaborative Fantasy Play and Storytelling. In Proceedings of CSCL 1999 (California, United States). DOI= http://web.media.mit.edu/ kimiko/publications.htm. 
[6] Segen, J. and Kumar, S. 1998. Video-based gesture interface to interactive movies. In Proceedings of the Sixth ACM international Conference on Multimedia: Technologies For interactive Movies (Bristol, United Kingdom, September 13 16, 1998). MULTIMEDIA '98. ACM Press, New York, NY, 39-42. DOI= http://doi.acm.org/10.1145/306774.306786

[7] Stanton, D., Neale, H., \& Bayon, V. (2002). Interfaces to support children's co-present collaboration: Multiple mice and tangible technologies. In Proceedings of CSCL 2002. 342-352. DOI =

http://citeseer.ist.psu.edu/cache/papers/cs/25944/http:zSzzSz
newmedia.colorado.eduzSzcsclzSz117.pdf/interfaces-tosupport-children.pdf.

[8] Stanton, D., Bayon, V., Neale, H., Ghali, A., Benford, S., Cobb, S., Ingram, R., O'Malley, C., Wilson, J., and Pridmore, T. 2001. Classroom collaboration in the design of tangible interfaces for storytelling. In Proceedings of the SIGCHI Conference on Human Factors in Computing Systems (Seattle, Washington, United States). CHI '01. ACM Press, New York, NY, 482-489. DOI= http://doi.acm.org/10.1145/365024.365322 